\title{
Evaluation of Decay Times from Noisy Room Responses with Pure-Tone Excitation
}

\author{
Mirosław MEISSNER \\ Institute of Fundamental Technological Research, Polish Academy of Sciences \\ Pawińskiego 5B, 02-106 Warszawa, Poland; e-mail: mmeissn@ippt.pan.pl \\ (received September 18, 2012; accepted December 24, 2012)
}

\begin{abstract}
Reverberant responses are widely used to characterize acoustic properties of rooms, such as the early decay time (EDT) and the reverberation times $T_{20}$ and $T_{30}$. However, in real conditions a sound decay is often deformed by background noise, thus a precise evaluation of decay times from noisy room responses is the main problem. In this paper this issue is examined by means of numerical method where the decay times are estimated from the decay function that has been determined by nonlinear polynomial regression from a pressure envelope obtained via the discrete Hilbert transform. In numerical experiment the room responses were obtained from simulations of a sound decay for two-room coupled system. Calculation results have shown that background noise slightly affects the evaluation of reverberation times $T_{20}$ and $T_{30}$ as long as the signal-to-noise ratio (SNR) is not smaller than about 25 and 35 dB, respectively. However, when the SNR is close to about 20 and $30 \mathrm{~dB}$, high overestimation of these times may occur as a result of bending up of the decay curve during the late decay.
\end{abstract}

Keywords: room acoustics, reverberation, decay times, room response, background noise, coupled rooms.

\section{Introduction}

One of the most fundamental aims of room acoustics is a prediction of decay times from measurements of a sound pressure decay inside enclosures. An accurate determination of decay times is primary for both absorption measurements in reverberation chambers (BARron, Coleman, 2001; NutTer et al., 2007) and the evaluation of acoustics of performance spaces (Bradley, 2005; Gota, SuderDęBska, 2009; Adelman-Larsen et al., 2010; BeRANEK, 2011) as well as ordinary rooms (DíAz, PEDRERO, 2005, 2007). Decay times are evaluated from a decay curve defined as the graphical representation of the decay of the sound pressure level in a room as a function of time after the cut-off of a continuous sound source (ISO 3382, 2012). The estimation of decay times is achieved by approximation of appropriate parts of the decay curve by fitting lines obtained by a linear least-squares regression and then a calculation of the decay times from the slope of these lines. Another method of determining the decay curve consists in the reverse-time integration of squared impulse response (Schroeder, 1965). This method results in exceptionally smooth decay curves, making a determi- nation of decay times simple and accurate. However, when the room impulse response is contaminated with high level background noise the method's accuracy is substantially reduced because of a distortion of decay curve slope during the late decay. This problem has been extensively studied in the past (CHU, 1978; Lundeby et al., 1995; XIANG, 1995; Morgan, 1997; Xiang, Goggans, 2001) and different remedial techniques have been proposed (KARJALAINEN et al., 2002; DragonetTi et al., 2009).

A subject of this paper is to study an accuracy of evaluation of decay times from room responses contaminated with background noise. The research is dedicated to low-frequency range where acoustic modes excited inside an enclosure are well separated. The reverberant response of room, which begins just after turning off the continuous sound source, is described theoretically by means of a modal expansion of a sound pressure for room systems with relatively small sound damping. The irregular room, which is considered in the study, has a form of the coupled room system consisting of two rectangular subrooms connected through an acoustically transparent opening. The choice of such a system was dictated by the fact that coupled room systems have the ability to create a nonlinear profile 
of pressure level decay (XIANG, GogGans, 2001, 2003; Meissner, 2007a, 2008a). The decay times were estimated from a decay function computed by nonlinear polynomial regression, corresponding to average longtime changes in a pressure envelope obtained via the discrete Hilbert transform. The practical issues in using the discrete Hilbert transform in signal processing were examined recently by the author (MEISSNER, $2012 \mathrm{a}, 2012 \mathrm{~b}$ ) and in order to improve an accuracy of this procedure, a numerical algorithm consisted in the appropriate modification of a discrete pressure signal, was proposed.

\section{Simulation of reverberant response of room}

In low-frequency range, the reverberant behaviour of room is strongly frequency dependent (MEISSNER, $2007 \mathrm{~b}, 2008 \mathrm{~b})$. Thus, in a theoretical model it was assumed that a room is excited by a a pure-tone sound source. When a steady-state is achieved, the source is abruptly switched off and the sound energy accumulated inside the room interior is absorbed on walls and an acoustic reverberation takes place. This reverberation consists of carrier waveforms of the decaying envelope that can be described by exponentially decreasing cosinusoidal functions (MEISSNER, 2008b)

$$
P_{m}(\mathbf{r}, t)=A_{m}(\mathbf{r}) e^{-r_{m} t} \cos \left(\Omega_{m} t-\beta_{m}\right),
$$

where $t \geq 0$ is the time, $\mathbf{r}=(x, y, z)$ represents the receiving position, $m=1,2,3, \ldots$ is the mode number, $A_{m}(\mathbf{r})$ describes the space distribution of mode amplitude

$$
A_{m}(\mathbf{r})=\frac{c^{2} \omega_{m} \Phi_{m}(\mathbf{r})\left[\int_{V} Q\left(\mathbf{r}^{\prime}\right) \Phi_{m}\left(\mathbf{r}^{\prime}\right) \mathrm{d} v^{\prime}\right]}{\Omega_{m} \sqrt{\left[\left(\omega_{m}^{2}-\omega^{2}\right)^{2}+4 r_{m}^{2} \omega^{2}\right]}},
$$

where $c$ is the sound speed, $V$ is the room volume, $\omega$ is the source frequency, $Q(\mathbf{r})$ is the volume source distribution, $\Phi_{m}(\mathbf{r})$ is the eigenfunction, $\omega_{m}$ is the natural mode frequency, $\Omega_{m}=\sqrt{\omega_{m}^{2}-r_{m}^{2}}$ is the mode frequency for damped oscillation, $\beta_{m}$ is the initial mode phase

$$
\beta_{m}=\tan ^{-1}\left[\frac{r_{m}\left(\omega_{m}^{2}+\omega^{2}\right)}{\Omega_{m}\left(\omega_{m}^{2}-\omega^{2}\right)}\right]
$$

and $r_{m}$ is the modal damping coefficient

$$
r_{m}=\frac{1}{2} \rho c^{2} \int_{S} \frac{\Phi_{m}^{2}\left(\mathbf{r}^{\prime}\right) \mathrm{d} s^{\prime}}{Z},
$$

where $\rho$ is the air density, $S$ is the surface of room walls and $Z$ is the wall impedance. In real conditions, the room response is usually deformed by the background noise, then assuming that the noise has a uniform spectral distribution, the formula for the reverberant response of room in noisy environment can be written as

$$
p(\mathbf{r}, t)=\sum_{m=1}^{M} P_{m}(\mathbf{r}, t)+A_{\mathrm{N}} \xi(t),
$$

where $A_{\mathrm{N}}$ is the noise amplitude and $\xi(t)$ is the unitylevel random signal. In a computer algorithm, the signal $\xi(t)$ is created by the function generating random real numbers from the range $(-1,1)$. In Eq. (5) the index $M$ corresponds to the last mode whose frequency is smaller than the Schroeder frequency

$$
f_{\mathrm{s}}=c \sqrt{\frac{6}{\mathcal{A}}},
$$

where $\mathcal{A}$ is the equivalent absorption area. As was proved by Schroeder (1996), below this frequency the modal density is low and particular modes can be decomposed from the room response, thus in multimode resonance systems the Schroeder frequency $f_{\mathrm{s}}$ marks the transition from individual, well-separated resonances to many overlapping modes.

An accuracy of the method of determining the decay times has been investigated for a coupled room system consisting of two connected rectangular subrooms. This was motivated by the fact that such a room is capable of producing a nonlinear profile of pressure level decay which may result in significant differences in decay times in the initial and late stages of sound decay. A horizontal cross-section of the room is shown in Fig. 1. The subrooms have the same height $h$ of $3 \mathrm{~m}$ and their lengths and widths are the following:

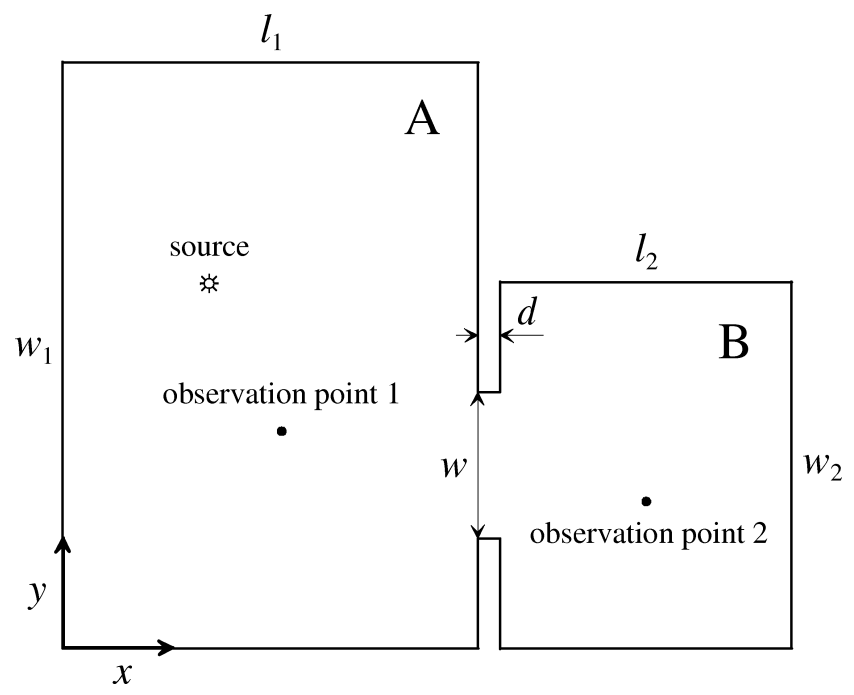

Fig. 1. Horizontal plan view of analysed room system consisting of two connected rectangular subrooms A and B. Symbols show positions of sound source and observation points. 
$l_{1}=5.7 \mathrm{~m}, l_{2}=4 \mathrm{~m}, w_{1}=8 \mathrm{~m}$ and $w_{2}=5 \mathrm{~m}$. The coupling between subrooms is realized by the opening having the height $h$, the width $w$ of $2 \mathrm{~m}$ and the thickness $d$ of $0.3 \mathrm{~m}$. The room system was excited with the power of $10^{-3} \mathrm{~W}$ by a harmonic source situated at the point: $x=2 \mathrm{~m}, y=5 \mathrm{~m}, z=1 \mathrm{~m}$. The room response was received at two observation points: $x=3 \mathrm{~m}, y=3 \mathrm{~m}, z=1.8 \mathrm{~m}$ (subroom A) and $x=8 \mathrm{~m}, y=2 \mathrm{~m}, z=1.8 \mathrm{~m}$ (subroom $\mathrm{B}$ ). The walls of subrooms A and B were assumed to be covered by materials having the random-absorption coefficients $\alpha_{1}$ and $\alpha_{2}$. Thus, the equivalent absorption area $\mathcal{A}$ is the following

$$
\mathcal{A}=\alpha_{1} S_{1}+\alpha_{2} S_{2},
$$

where $S_{1}$ and $S_{2}$ are surfaces of walls in subrooms A and B. In a numerical simulation the coefficients $\alpha_{1}$ and $\alpha_{2}$ were set to 0.04 and 0.185 , respectively. Therefore, in the considered case a sound absorption in subroom A was much smaller than in subroom B. Using Eqs. (6) and (7), and the assumed values $\alpha_{1}$ and $\alpha_{2}$, it is easy to calculate that the Schroeder frequency is as follows: $f_{\mathrm{s}} \approx 174 \mathrm{~Hz}$. Below this frequency 150 eigenmodes were found and for this set of modes the reverberant responses of room in receiving positions were simulated. The eigenfunctions $\Phi_{m}$ were computed by a numerical solution of the wave equation where the finite difference method and the forced oscillator method were employed (MEISSNER, 2007b).

A computer reconstruction of room reverberant response was performed for two source frequencies: 107 and $151 \mathrm{~Hz}$, which correspond approximately to eigenfrequencies of 44th and 107th modes. The room responses computed for these frequencies under noisefree conditions are shown in Fig. 2. Calculation results indicate in a clear way that in the analyzed coupled room system a shape of the room response and an initial amplitude of a sound decay strongly depend on the sound frequency and a position of the observation point. For example, when the source frequency is set to $107 \mathrm{~Hz}$, a smooth decay of the sound pressure is observed inside the subroom A in the first receiving position (Fig. 2a). However, a shift of this position to the subroom $\mathrm{B}$ results in a fundamental change of the response consisting in a rapid decrease in a pressure in the initial stage of a sound decay (Fig. 2b). On the other hand, for the source frequency of $151 \mathrm{~Hz}$ large wave fluctuations in reverberant responses are noted suggesting that in these cases the beating effect is present (Figs. 2c, d).
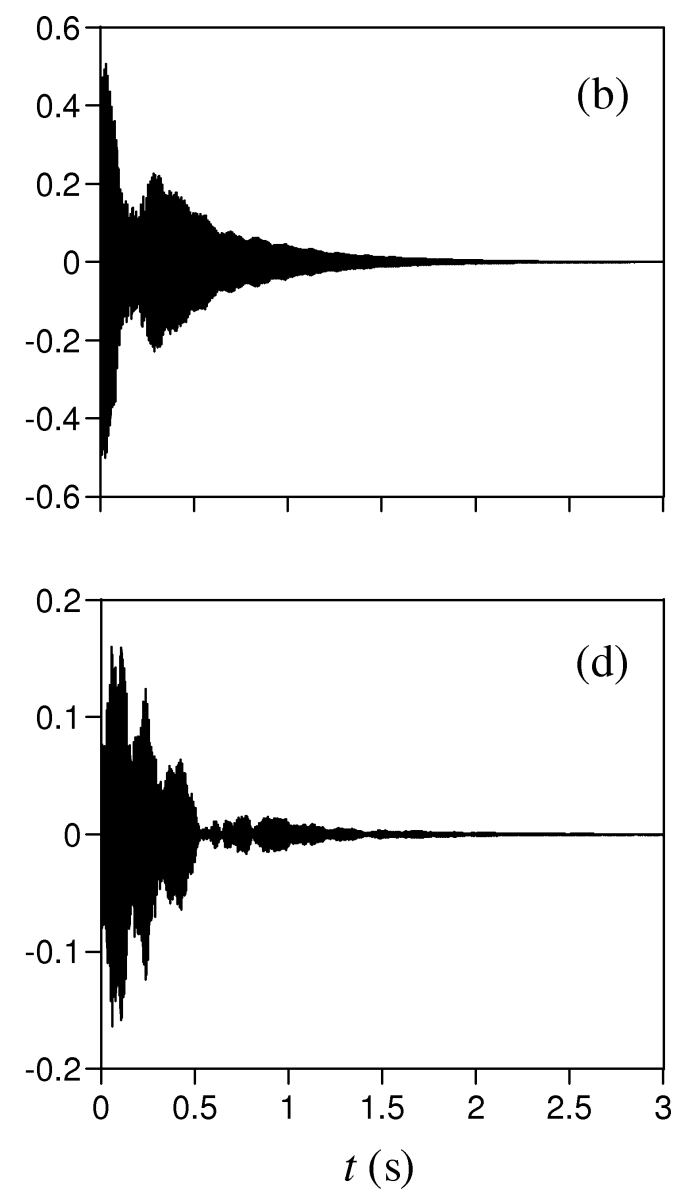

Fig. 2. Temporal changes in sound pressure $p$ for source frequencies: a), b) $107 \mathrm{~Hz}$ and c), d) $151 \mathrm{~Hz}$, and noise-free conditions. Position of observation point: a), c) subroom A, b), d) subroom B. 


\section{Detection of response envelope via discrete Hilbert transform}

The Hilbert transform is an important tool for a signal analysis because it can be used in a direct examination of instantaneous properties of the signal such as an envelope and a phase. An application of this method to the reverberant response of rooms enables a detection of the response envelope giving a more accurate prediction of decay times. In continuous time domain a classical definition of the Hilbert transform $\mathcal{H}$ is as follows (HAHN, 1996)

$$
\mathcal{H}[s(t)]=\frac{1}{\pi} \int_{-\infty}^{\infty} \frac{s(\tau)}{t-\tau} \mathrm{d} \tau,
$$

where $s(t)$ is a real-valued signal and the symbol $f$ denotes the principal value integral because of the possible singularity at $\tau=t$. Of course, the room response obtained by a numerical simulation is of finite length and digitally sampled, thus if the pressure $p(\mathbf{r}, t)$ from Eq. (5) is uniformly sampled with the period $T$, the discrete-time pressure signal is obtained

$$
p[n]=p(\mathbf{r}, t[n])
$$
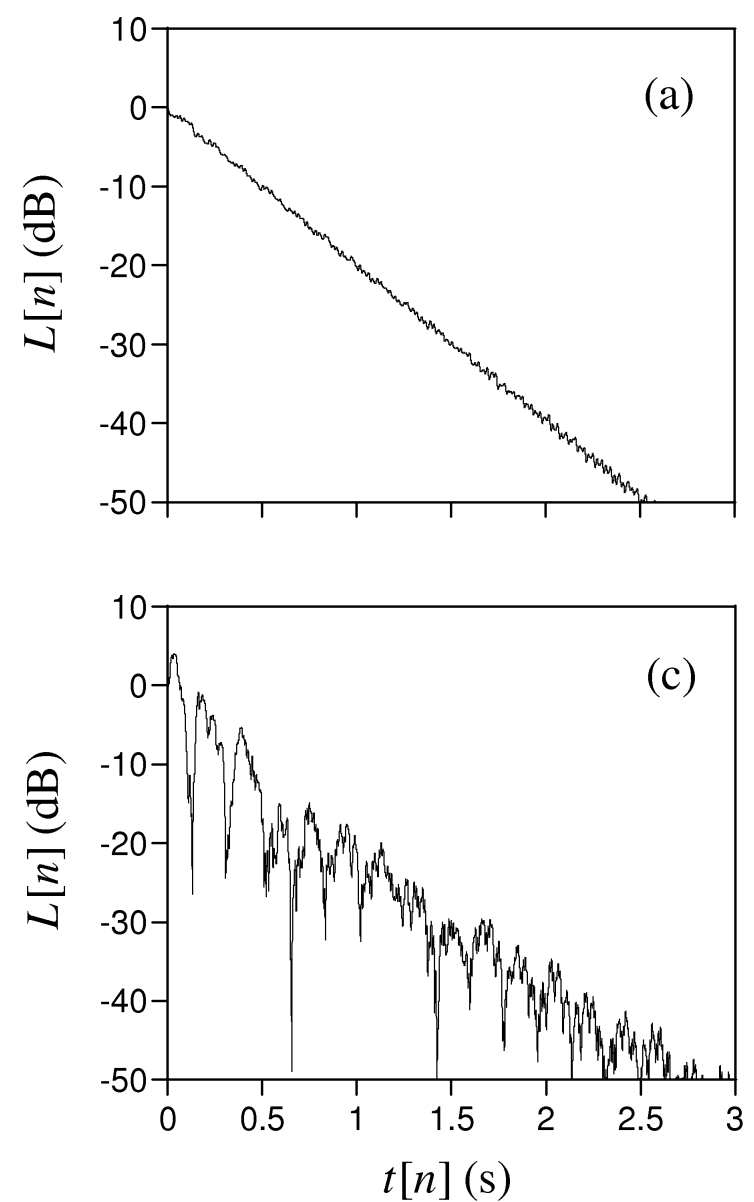

where $t[n]=n T, n=0,1, \ldots, N$ and on the left side, the spatial coordinate $\mathbf{r}$ is omitted for simplicity of notation. In discrete time case, the Hilbert transform $\mathcal{H}$ is replaced by the discrete Hilbert transform $\mathcal{H}_{d}$. In order to improve an exactness of the discrete Hilbert transform in the prediction of pressure envelope the extended discrete signal $P[n]$ determined on the basis of the original pressure signal $p[n]$ is introduced (MEIssNER, 2012a)

$$
P[n]= \begin{cases}-p[N-n], & n=0,1, \ldots, N-1, \\ p[-N+n], & n=N, N+1, \ldots, 2 N .\end{cases}
$$

The new discrete signal is determined in a doubleextended time interval and represents a discrete function having a rotational symmetry with respect to the origin of coordinate system. This method of signal processing reduces an inaccuracy of the discrete Hilbert transform generated by a limitation of a signal duration (end effect) and caused by the fact that for exponentially decaying harmonic signals the Bedrosian identity (BEDROSIAN, 1963) is not satisfied. Using a definition of the discrete Hilbert transform for nonperiodic signals (KAK, 1970), the Hilbert transform of the extended signal $P[n]$ is determined by
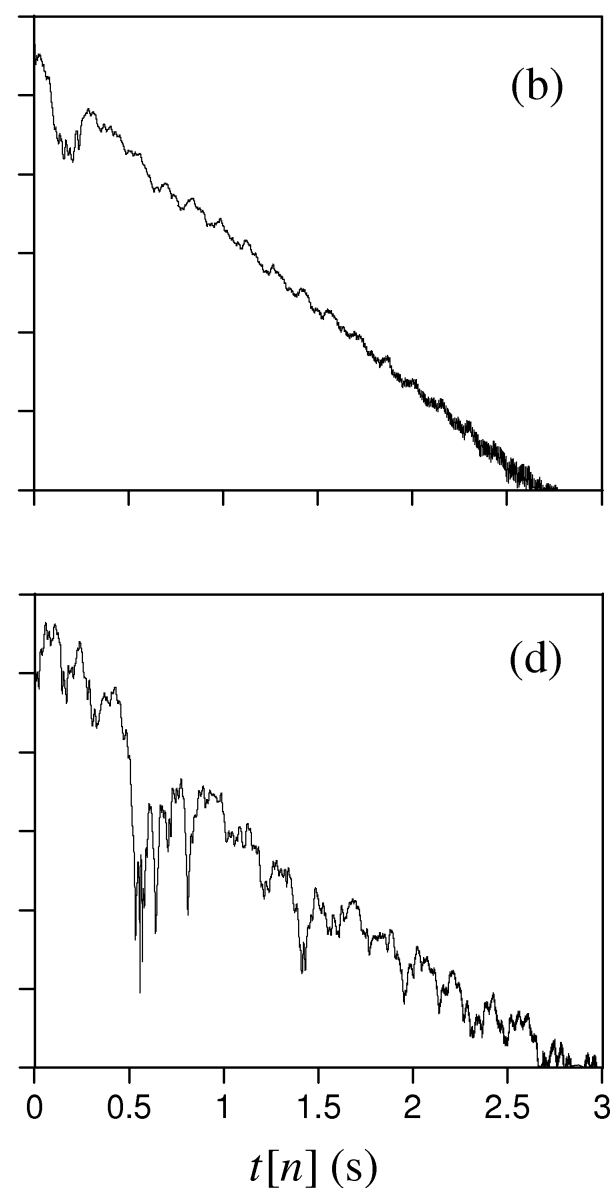

Fig. 3. Temporal decay of level $L[n]$ of pressure envelope for signals shown in Fig. 2. 


$$
\mathcal{H}_{d}\{P[n]\}= \begin{cases}\frac{2}{\pi} \sum_{m=\text { odd }} \frac{P[m]}{n-m}, & n \text { even, } \\ \frac{2}{\pi} \sum_{m=\text { even }} \frac{P[m]}{n-m}, & n \text { odd }\end{cases}
$$

Subsequently, a logarithmic decay of a pressure envelope is found from the equation

$$
L[n]=20 \log (E[n] / E[0]),
$$

where $E[n]=\sqrt{P^{2}[n]+\mathcal{H}_{d}^{2}\{P[n]\}}$ and $E[0]$ is the envelope at a beginning of a sound decay.

Calculation results in Fig. 3 depict temporal changes in the relative level $L[n]$ of the pressure envelope calculated for the room responses shown in Fig. 2 by applying the method presented above. For the first room response, the level $L[n]$ in the analysed time domain decreases almost linearly with the time showing that in this case a reverberation process can be described by a single decay time. A characteristic property of the second room response is a considerable difference between decay times in the early and late stages of the decaying sound. In the last two cases significant fluctuations of the level $L[n]$ are observed and they are due to a presence of two dominant modes of slightly different frequencies in room responses. This causes the pressure envelope to fluctuate with a frequency equal to the difference between frequencies of these modes (the beating effect).

Numerical data in Fig. 4 show changes in the room responses when the sound decay is deformed by a high level background noise $\left(A_{\mathrm{N}}=2 \times 10^{-2} \mathrm{~Pa}\right.$, the noise level $L_{\mathrm{N}}$ of $60 \mathrm{~dB}$ ). Under such very noisy conditions, the decrease in the relative level $L[n]$ is noted in the initial stage of reverberation process until the decaying signal is masked by the noise. A duration of this initial stage depends directly on the signal-to-noise ratio (SNR) and of course, it is visibly smaller for low SNRs as is evident from the comparison of Figs. 2 and 4.

\section{Evaluation of decay times}

As was shown in the previous section, an application of the discrete Hilbert transform method is a simple way to calculate the relative level $L[n]$ of a pressure envelope in room responses. However, an accuracy of a direct evaluation of decay times from changes in the level $L[n]$ is substantially limited by a presence of background noise and large fluctuations of pressure in some responses. Therefore, in a numerical procedure
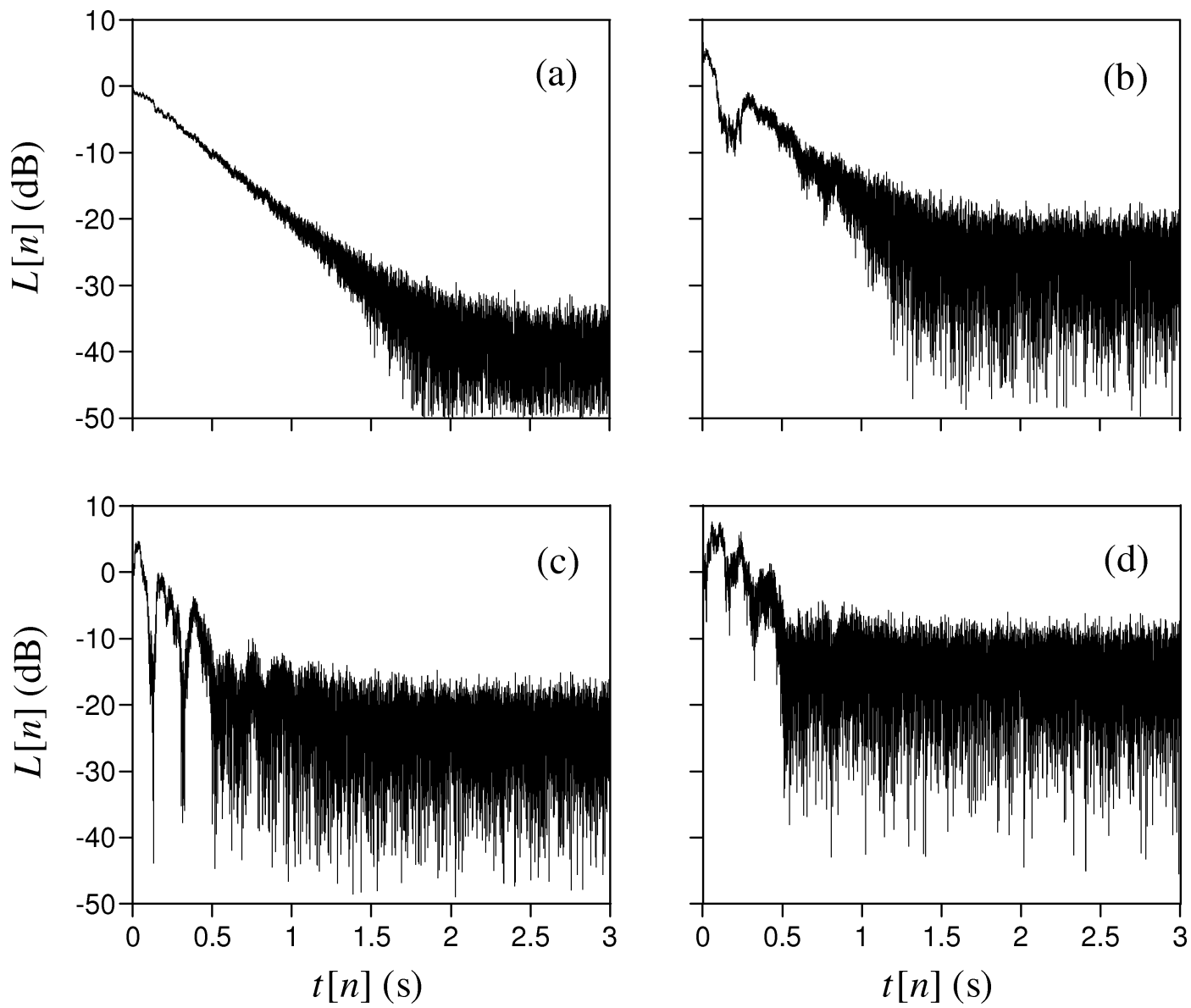

Fig. 4. Temporal changes in level $L[n]$ for signals from Fig. 2 with added high level background noise $\left(L_{\mathrm{N}}=60 \mathrm{~dB}\right)$. 
the function $L[n]$ was employed to construct the decay function $L_{\mathrm{av}}$ describing average long-time changes in a sound pressure level. This function was calculated by nonlinear polynomial regression and it was finally used to predict decay times. Examples of functions $L_{\mathrm{av}}$ computed by this method (solid lines) and the level $L[n]$ corresponding to these functions (gray lines) are shown in Fig. 5. The results were obtained for the sound source of frequency $151 \mathrm{~Hz}$ located in the subroom A and the background noise level $L_{\mathrm{N}}$ of 0 and $60 \mathrm{~dB}$.
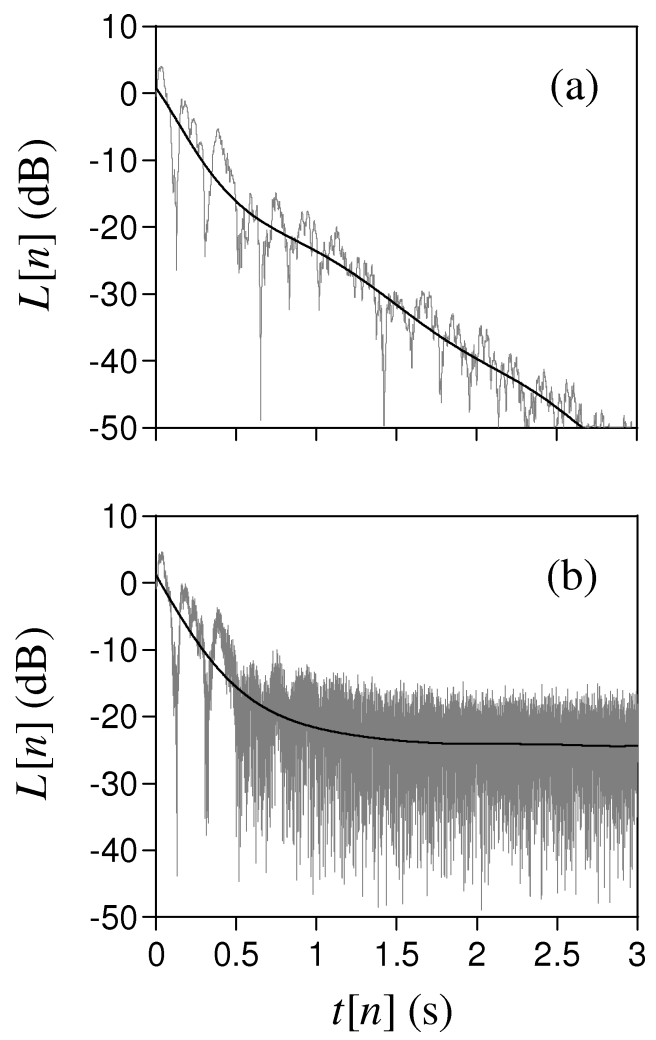

Fig. 5. Level $L[n]$ of pressure envelope (gray lines) and decay function $L_{\mathrm{av}}$ calculated by polynomial regression of 8th order (solid lines) for source frequency of $151 \mathrm{~Hz}$ and noise level $L_{\mathrm{N}}$ : a) $0 \mathrm{~dB}$, b) $60 \mathrm{~dB}$. Observation point in subroom $\mathrm{A}$.

The estimation of decay times was based on finding a fit line to appropriate parts of the function $L_{\mathrm{av}}$ and it was realized by a linear regression. In order to properly characterize the reverberation process, three decay times were computed: the early decay time (EDT) predicted on the basis of a drop of $L_{\mathrm{av}}$ from 0 to $-10 \mathrm{~dB}$ and the reverberation times $T_{20}$ and $T_{30}$ estimated from a decrease in $L_{\mathrm{av}}$ from -5 to -25 and $-35 \mathrm{~dB}$, respectively. The early decay time is always determined from measurements because the initial decay is important from the subjective viewpoint. The decay time $T_{30}$ is a standard measure of the reverberation time under low-noise conditions. However, when the decay curve does not have sufficient dynamic range due to the presence of high level background noise, the de- cay time $T_{20}$ is used as appropriate measures of the reverberation time (ISO 3382, 2012).

Results of evaluation of the decay times EDT, $T_{20}$ and $T_{30}$ are summarized in Tables 1 and 2 . They illustrate changes in the decay times with the background noise level $L_{\mathrm{N}}$ increasing from 0 to $60 \mathrm{~dB}$ in $10 \mathrm{~dB}$ steps and show variations of these times with corresponding SNRs. In addition to these data, in Tables 1 and 2 dimensionless parameters $\Delta_{\mathrm{EDT}}, \Delta_{T_{20}}$ and $\Delta_{T_{30}}$ are also collected. These quantities are defended as

$$
\begin{aligned}
\Delta_{\mathrm{EDT}} & =\frac{\left|\operatorname{EDT}\left(L_{\mathrm{N}}\right)-\operatorname{EDT}(0)\right|}{\operatorname{EDT}(0)}, \\
\Delta_{T_{20}} & =\frac{\left|T_{20}\left(L_{\mathrm{N}}\right)-T_{20}(0)\right|}{T_{20}(0)}, \\
\Delta_{T_{30}} & =\frac{\left|T_{30}\left(L_{\mathrm{N}}\right)-T_{30}(0)\right|}{T_{30}(0)},
\end{aligned}
$$

where $\operatorname{EDT}(0), T_{20}(0)$ and $T_{30}(0)$ are values of decay times for the background noise level $L_{\mathrm{N}}$ equal to zero $\left(A_{\mathrm{N}}=2 \times 10^{-5} \mathrm{~Pa}\right)$, thus they represent relative errors in the evaluation of decay times due to the presence of background noise.

Simulation data collected in upper part of Table 1 were obtained in the receiving position located in the subroom A for the source frequency of $107 \mathrm{~Hz}$. In this case the room response is characterized by large and moderate SNRs (37-97 dB) resulting in a good accuracy in determination of decay times. Since the response is dominated by one acoustic mode all decay times are very similar $(3.012-3.050 \mathrm{~s})$. For the same source frequency and the observation point located in subroom B, the decay function exhibits a "sagging" appearance in an initial stage of decay (Fig. 3) causing that the early decay time is approximately three times smaller than the reverberation times $T_{20}$ and $T_{30}$ evaluated from the late decay. Horizontal lines in Table 1 denote the case when the SNR is below $30 \mathrm{~dB}$ making it impossible to determine the reverberation time $T_{30}$.

Calculation results in Table 2 were obtained for the source frequency of $151 \mathrm{~Hz}$. They confirm that decay functions created on the basis of signals received at both observation points are highly nonlinear because the decay times considerably differ. For example, if the observation point is located in the subroom $\mathrm{A}$ and the background noise level $L_{\mathrm{N}}$ is zero, the early decay time is approximately 1.9 times smaller than $T_{20}$ and 2.2 times smaller than $T_{30}$. On the other hand, when this point is located in the subroom $\mathrm{B}$, the ratios $T_{20} / \mathrm{EDT}$ and $T_{30} / \mathrm{EDT}$ are respectively equal to 2.6 and 2.3 for $L_{\mathrm{N}}=0$. Because of smaller signal dynamics, the effect of background noise on the reverberation process manifests itself through high increase in reverberation times $T_{20}$ and $T_{30}$ for the lowest SNRs (observation point in subroom A) or a total impossibility of determining these reverberation times for a number of cases (observation point in subroom B). 
Table 1. Decay times EDT, $T_{20}, T_{30}$ and relative errors $\Delta_{\mathrm{EDT}}, \Delta_{T_{20}}, \Delta_{T_{30}}$ versus background noise level $L_{\mathrm{N}}$ and signal-to-noise ratio. Source frequency of $107 \mathrm{~Hz}$. Observation point in subroom A (upper part of table) and subroom B (bottom part of table).

\begin{tabular}{|c|c|c|c|c|c|c|c|}
\hline$L_{\mathrm{N}}[\mathrm{dB}]$ & $\mathrm{SNR}[\mathrm{dB}]$ & $\mathrm{EDT}[\mathrm{s}]$ & $\Delta_{\mathrm{EDT}}[\%]$ & $T_{20}[\mathrm{~s}]$ & $\Delta_{T_{20}}[\%]$ & $T_{30}[\mathrm{~s}]$ & $\Delta_{T_{30}}[\%]$ \\
\hline 0 & 97 & 3.050 & 0 & 3.012 & 0 & 3.028 & 0 \\
10 & 87 & 3.050 & 0.006 & 3.012 & 0.002 & 3.028 & 0 \\
20 & 77 & 3.049 & 0.024 & 3.012 & 0.009 & 3.028 & 0.001 \\
30 & 67 & 3.047 & 0.080 & 3.012 & 0.031 & 3.028 & 0.003 \\
40 & 57 & 3.048 & 0.071 & 3.013 & 0.052 & 3.028 & 0.007 \\
50 & 47 & 3.026 & 0.782 & 3.015 & 0.107 & 3.033 & 0.179 \\
60 & 37 & 3.035 & 0.497 & 3.025 & 0.456 & 3.029 & 0.047 \\
\hline 0 & 88 & 1.086 & 0 & 3.498 & 0 & 3.260 & 0 \\
10 & 78 & 1.089 & 0.237 & 3.498 & 0.013 & 3.260 & 0.007 \\
20 & 68 & 1.097 & 0.959 & 3.498 & 0.024 & 3.260 & 0.026 \\
30 & 58 & 1.105 & 1.712 & 3.497 & 0.055 & 3.258 & 0.057 \\
40 & 48 & 1.117 & 2.861 & 3.495 & 0.109 & 3.259 & 0.016 \\
50 & 38 & 1.186 & 9.179 & 3.490 & 0.236 & 3.245 & 0.463 \\
60 & 28 & 1.240 & 14.12 & 3.460 & 1.099 & - & - \\
\hline
\end{tabular}

Table 2. Decay times EDT, $T_{20}, T_{30}$ and relative errors $\Delta_{\mathrm{EDT}}, \Delta_{T_{20}}, \Delta_{T_{30}}$ versus background noise level $L_{\mathrm{N}}$ and signal-to-noise ratio. Source frequency of $151 \mathrm{~Hz}$. Observation point in subroom A (upper part of table) and subroom B (bottom part of table).

\begin{tabular}{|c|c|c|c|c|c|c|c|}
\hline$L_{\mathrm{N}}[\mathrm{dB}]$ & $\mathrm{SNR}[\mathrm{dB}]$ & EDT $[\mathrm{s}]$ & $\Delta_{\mathrm{EDT}}[\%]$ & $T_{20}[\mathrm{~s}]$ & $\Delta_{T_{20}}[\%]$ & $T_{30}[\mathrm{~s}]$ & $\Delta_{T_{30}}[\%]$ \\
\hline 0 & 81 & 1.554 & 0 & 2.889 & 0 & 3.451 & 0 \\
10 & 71 & 1.554 & 0.041 & 2.888 & 0.055 & 3.451 & 0.003 \\
20 & 61 & 1.554 & 0.008 & 2.890 & 0.011 & 3.452 & 0.024 \\
30 & 51 & 1.569 & 0.949 & 2.942 & 1.830 & 3.463 & 0.352 \\
40 & 41 & 1.584 & 1.907 & 3.002 & 3.909 & 3.490 & 1.123 \\
50 & 31 & 1.604 & 3.175 & 3.118 & 7.926 & 6.655 & 92.84 \\
60 & 21 & 1.537 & 1.080 & 5.501 & 90.38 & - & - \\
\hline 0 & 72 & 1.631 & 0 & 4.246 & 0 & 3.818 & 0 \\
10 & 62 & 1.631 & 0.041 & 4.249 & 0.070 & 3.818 & 0.012 \\
20 & 52 & 1.630 & 0.050 & 4.255 & 0.199 & 3.823 & 0.153 \\
30 & 42 & 1.613 & 1.070 & 4.276 & 0.693 & 3.863 & 1.192 \\
40 & 32 & 1.589 & 2.527 & 4.334 & 2.071 & - & - \\
50 & 22 & 1.687 & 3.478 & - & - & - & - \\
60 & 12 & 2.003 & 22.84 & - & - & - & - \\
\hline
\end{tabular}

\section{Summary and conclusions}

Reverberation is the most basic and easily perceived acoustical property of enclosures, therefore one of the most important objectives of room acoustics is an evaluation of decay times from room reverberant responses. However, in real measurements a late sound decay is usually contaminated with background noise, thus an accurate prediction of reverberation times from noisy room responses is the main concern. The problem was examined using a numerical technique where the decay times are estimated from the best straight line fit to the decay function that has been calculated by nonlinear polynomial regression from a pressure envelope obtained via the discrete Hilbert transform. A choice of such a procedure was a consequence of large fluctuations of the pressure envelope arising from noise disturbances and modal interactions which cause the beating effect.

Room responses exploited in a numerical experiment were adopted from sound decay simulations, performed for a coupled room system consisting of two connected rectangular subrooms. As a result of complex room shape and irregular distribution of absorbing material, a nonlinear behaviour of a pressure level decay was noted. The numerical experiment indicated that the background noise only slightly influences the evaluation of reverberation times $T_{20}$ and $T_{30}$ as long as the SNR is not smaller than about 25 and $35 \mathrm{~dB}$, respectively. This implies that the proposed method 
tolerates clearly smaller dynamic ranges for evaluation of the reverberation times $T_{20}$ and $T_{30}$ than is required by the ISO 3382 standard.

In the past, several method were applied for estimating decay times. A nonlinear iterative regression method for evaluating reverberation times from Schroeder's decay curves was proposed by XIANG (1995). He found that the SNR of $35 \mathrm{~dB}$ is a sufficient dynamic range for accurate prediction of the reverberation time $T_{30}$ and this result is in accordance with the finding of this study. The method of XIANG was extended by XIANG and Goggans (2001) to multirate decay functions using Bayesian probability theory and, as was demonstrated, for two coupled rooms Bayesian decay time estimation yields a reliable value when the SNR is higher than $41 \mathrm{~dB}$.

\section{References}

1. Adelman-Larsen N., Thompson E., Gade A. (2010), Suitable reverberation times for halls for rock and pop music, Journal of the Acoustical Society of America, 127, 1, 247-255.

2. Barron M., Coleman S. (2001), Measurements of the absorption by auditorium seating - a model study, Journal of Sound and Vibration, 239, 4, 573-587.

3. Bedrosian E. (1963), A product theorem for Hilbert transform, Proceedings of the IEEE, 51, 5, 868-869.

4. BerAneK L. (2011), Concert hall acoustics, Architectural Science Review, 54, 1, 5-14.

5. BRADLEY J. (2005), Using ISO 3382 measures, and their extensions, to evaluate acoustical conditions in concert halls, Acoustical Science and Technology, 26, 2, 170-178.

6. CHu W. (1978), Comparison of reverberation measurements using Schroeder's impulse method and decaycurve averaging method, Journal of the Acoustical Society of America, 63, 5, 1444-1450.

7. Díaz C., Pedrero A. (2005), The reverberation time of furnished rooms in dwellings, Applied Acoustics, 66, 8, 945-956.

8. Díaz C., Pedrero A. (2007), The reverberation time and equivalent sound absorption area of rooms in dwellings, Noise and Vibration Worldwide, 38, 6, 1220.

9. Dragonetti R., Ianniello C., Romano R. (2009), Reverberation time measurement by the product of two room impulse responses, Applied Acoustics, 70, 1, 231243.

10. GołA A., Suder-DęBSKA K. (2009), Analysis of Dome Home Hall theatre acoustic field, Archives of Acoustics, 34, 3, 273-293.

11. Hahn S. (1996), The Hilbert transforms in signal processing, Artech House Inc., Boston.

12. ISO 3382 (2012), Acoustics - Measurement of room acoustic parameters. Part 1: Performance spaces. Part 2: Reverberation time in ordinary rooms, International Organization for Standardization, Géneve.
13. KAK S. (1970), The discrete Hilbert transform, Proceedings of the IEEE, 58, 4, 585-586.

14. Karjalainen M., Antsalo P., Mäkivirta A., PelTONEN T., VÄLIMÄKI V. (2002), Estimation of modal decay parameters from noisy response measurements, Journal of the Audio Engineering Society, 50, 11, 867878.

15. Lundeby A., Vigran T., Bietz H., Vorländer M. (1995), Uncertainties of measurements in room acoustics, Acustica, 81, 4, 344-355.

16. MeIssner M. (2007a), Analysis of non-exponential sound decay in an enclosure composed of two connected rectangular subrooms, Archives of Acoustics, 32, 4S, 213-220.

17. Meissner M. (2007b), Computational studies of steady-state sound field and reverberant sound decay in a system of two coupled rooms, Central European Journal of Physics, 5, 3, 293-312.

18. Meissner M. (2008a), Influence of absorbing material distribution on double slope sound decay in L-shaped room, Archives of Acoustics, 33, 4S, 159-164.

19. Meissner M. (2008b), Influence of wall absorption on low-frequency dependence of reverberation time in room of irregular shape, Applied Acoustics, 69, 7, 583-590.

20. Meissner M. (2012a), The discrete Hilbert transform and its application to the analysis of reverberant decay of modal vibrations in enclosures, Journal of Vibration and Control, 18, 11, 1595-1606.

21. Meissner M. (2012b), Accuracy issues of discrete Hilbert transform in identification of instantaneous parameters of vibration signals, Acta Physica Polonica A, 121, 1A, 164-167.

22. Morgan D. (1997), A parametric error analysis of the backward integration method for reverberation time estimation, Journal of the Acoustical Society of America, 101, 5, 2686-2693.

23. Nutter D., Leishman T., Sommerfeldt S., BlotTER J. (2007), Measurement of sound power and absorption in reverberation chambers using energy density, Journal of the Acoustical Society of America, 121, 5, 2700-2710.

24. Schroeder M. (1965), New method of measuring reverberation time, Journal of the Acoustical Society of America, 37, 3, 400-412.

25. Schroeder M. (1996), The "Schroeder frequency" revisited, Journal of the Acoustical Society of America, 99, 5, 3240-3241.

26. XiAng N. (1995), Evaluation of reverberation times using a nonlinear regression approach, Journal of the Acoustical Society of America, 98, 4, 2112-2121.

27. Xiang N., Goggans P. (2001), Evaluation of decay times in coupled spaces: Bayesian parameter estimation, Journal of the Acoustical Society of America, 110, $3,1415-1424$.

28. Xiang N., Goggans P. (2003), Evaluation of decay times in coupled spaces: Bayesian decay model selection, Journal of the Acoustical Society of America, 113, 5, 2685-2697. 